\title{
Liposomal Treatment of Experimental Arthritis Can Be Monitored Noninvasively with a Radiolabeled Anti-Fibroblast Activation Protein Antibody
}

\author{
Tessa van der Geest ${ }^{1}$, Peter Laverman ${ }^{1}$, Danny Gerrits ${ }^{1}$, Birgitte Walgreen ${ }^{2}$, Monique M. Helsen ${ }^{2}$, Christian Klein ${ }^{3}$, \\ Tapan K. Nayak ${ }^{4}$, Gert Storm ${ }^{5,6}$, Josbert M. Metselaar ${ }^{6,7}$, Marije I. Koenders ${ }^{2}$, and Otto C. Boerman ${ }^{1}$ \\ ${ }^{1}$ Department of Radiology and Nuclear Medicine, Radboud University Medical Center, Nijmegen, The Netherlands; ${ }^{2}$ Department of \\ Experimental Rheumatology, Radboud University Medical Center, Nijmegen, The Netherlands; ${ }^{3}$ Roche Pharmaceutical Research and \\ Early Development, Innovation Center Zurich, Schlieren, Switzerland; ${ }^{4}$ Roche Pharmaceutical Research and Early Development, \\ Innovation Center Basel, Basel, Switzerland; ${ }^{5}$ Department of Pharmaceutics, Utrecht Institute for Pharmaceutical Sciences, Utrecht \\ University, Utrecht, The Netherlands; ${ }^{6}$ Department of Targeted Therapeutics, MIRA Institute, University of Twente, Zuidhorst, The \\ Netherlands; and ${ }^{7}$ Department of Experimental Molecular Imaging, University Clinic and Helmholtz Institute for Biomedical \\ Engineering, RWTH Aachen University, Aachen, Germany
}

Rheumatoid arthritis is a chronic autoimmune disorder resulting in synovial inflammation. Fibroblast activation protein (FAP) is overexpressed by fibroblastlike synoviocytes in arthritic joints. Radioimmunoimaging with an anti-FAP antibody might be used to monitor the response to therapy, thus enabling tailored therapy strategies and therapeutic outcomes. The aim of this study was to assess whether a radiolabeled anti-FAP antibody could be used to monitor the efficacy of treatment with long-circulating liposomes (LCL) containing prednisolone phosphate (PLP-LCL) in a mouse model of arthritis. Methods: Collagen-induced arthritis $(\mathrm{CIA})$ was induced in male DBA $1 \mathrm{~J}$ mice. Mice were treated with a single injection $(10 \mathrm{mg} / \mathrm{kg})$ of PLP-LCL or empty LCL as a control. SPECT and CT images were acquired $24 \mathrm{~h}$ after injection of ${ }^{99 \mathrm{~m} T c-l a b e l e d ~ s u c c i n i m i d y l-}$

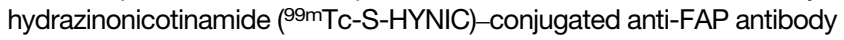
$28 \mathrm{H} 1$ at 2, 5, and $9 \mathrm{~d}$ after treatment. The uptake of $99 \mathrm{mTc}-\mathrm{S}-$ HYNIC-28H1 in all joints was quantified and correlated with macroscopic arthritis scores. Results: Treatment of CIA with PLP-LCL significantly suppressed joint swelling. At just $1 \mathrm{~d}$ after treatment, the macroscopic arthritis scores had decreased by $50 \%$. Scores decreased further, to only $10 \%$ of the initial scores, at 5 and $9 \mathrm{~d}$ after treatment. In contrast, macroscopic arthritis scores had increased up to $600 \%$ in untreated mice at $9 \mathrm{~d}$ after the injection of empty LCL. 99mTc-S-HYNIC-28H1 uptake ranged from 1.5 percentage injected dose per gram in noninflamed joints to 22.6 percentage injected dose per gram in severely inflamed joints. The uptake of radiolabeled $28 \mathrm{H} 1$ in inflamed joints (percentage injected dose) correlated with the arthritis score (Spearman $\rho, 0.77 ; P<0.0001)$. Moreover, the uptake of ${ }^{99 m T c}$-S-HYNIC-28H1 was slightly increased at $9 \mathrm{~d}$ after therapy but was not seen macroscopically, indicating that SPECT/CT imaging might be more sensitive than the macroscopic arthritis scoring method. Conclusion: SPECT/CT imaging with ${ }^{99 m T C-S-H Y N I C-~}$ $28 \mathrm{H} 1$ specifically monitored the response to therapy, and tracer accumulation correlated with the severity of inflammation. In addition, SPECT/CT imaging was potentially more sensitive than the macroscopic

Received May 8, 2016; revision accepted Jul. 13, 2016.

For correspondence or reprints contact: Tessa van der Geest, Department of Radiology and Nuclear Medicine, Radboud University Medical Center, P.O. Box 9101, 6500 HB Nijmegen, The Netherlands.

E-mail: Tessa.vanderGeest@radboudumc.nl

Published online Aug. 4, 2016.

COPYRIGHT (C) 2017 by the Society of Nuclear Medicine and Molecular Imaging. arthritis scoring method. This study showed that SPECT/CT with ${ }^{99 \mathrm{mTC}-}$ S-HYNIC-28H1 could be used to noninvasively monitor the course of CIA in mice.

Key Words: fibroblast activation protein; SPECT/CT imaging; collagen-induced arthritis; prednisolone phosphate-encapsulating PEG liposomes; therapy monitoring

J Nucl Med 2017; 58:151-155

DOI: 10.2967/jnumed.116.177931

$\mathbf{R}$ heumatoid arthritis (RA) is an autoimmune disorder that affects $0.5 \%-1 \%$ of the European population (1). It is characterized by inflammation of synovial joints and eventually leads to cartilage and bone destruction. At present, RA is treated mostly systemically (orally, intramuscularly, or intravenously) by combinations of nonsteroidal antiinflammatory drugs, disease-modifying antirheumatic drugs, biological agents, and corticosteroids or sometimes locally (intraarticular corticosteroid injections) $(2,3)$. All of these therapeutic treatments aim to relieve pain, reduce inflammation, and prevent irreversible damage. Unfortunately, therapeutic effects may take several months to occur, especially after treatment with diseasemodifying antirheumatic drugs; in a subpopulation of patients, nonresponsiveness to the drugs is seen. Therefore, there is a need for a noninvasive imaging method to objectively determine the severity of symptoms, to monitor therapeutic efficacy, and to predict the occurrence of exacerbations during the course of the disease. At an early stage after treatment, specific and sensitive therapy monitoring could allow for an alteration of the therapeutic strategy when therapeutic effects are shown to be insufficient, potentially decreasing the unwanted side effects of ineffective drugs. In addition, an early switch to an effective drug could protect patients from irreversible damage due to RA.

The synovium of RA patients contains high levels of inflammatory cells, including macrophages and fibroblastlike synoviocytes $(4,5)$. These cells are prominently present in areas with high inflammatory activity and have an aggressive, invasive character. When activated, macrophages produce proinflammatory cytokines, 
such as interleukin 1 and tumor necrosis factor $\alpha$, triggering the production of proinflammatory cytokines (6). After stimulation by interleukin 1 and tumor necrosis factor $\alpha$, fibroblastlike synoviocytes produce proinflammatory cytokines and proteolytic enzymes, such as interleukin 6 and matrix metalloproteinases $(7,8)$. These mediators stimulate and amplify inflammation, contribute to degradation of the extracellular matrix, and exacerbate joint damage. Patients are often treated with corticosteroids, such as prednisolone phosphate (PLP), to suppress inflammation and reduce the levels of proinflammatory cytokines. PLP reduces the accumulation of macrophages and irreversible cartilage damage $(9,10)$. To increase efficacy and reduce toxicity, PLP can be given locally or encapsulated in drug delivery systems. In experimental arthritis models, the use of long-circulating liposomes (LCL) to target all inflamed joints at once with one intravenous injection created local persisting depots of steroids and increasing ratios of efficacy to safety $(11,12)$.

Molecular imaging has already been proven successful in noninvasive and early response monitoring but, in most cases, is not specific for arthritis at a molecular level $(11,13)$. Fibroblast activation protein (FAP) is a cell surface-bound, type II transmembrane glycoprotein belonging to the family of serine prolyl oligopeptidases. In arthritic joints, FAP is dominantly expressed by activated fibroblastlike synoviocytes and is found in human arthritis as well as experimental arthritis, such as the collagen-induced arthritis (CIA) model (14). FAP plays a role in pannus formation and progression of disease in all stages (15). Antibody $28 \mathrm{H} 1$ is a noninternalizing antibody with a high affinity for human and murine FAP (16). The specificity of $28 \mathrm{H} 1$ was shown in an vitro binding assay and by immunohistochemistry in arthritic joints (16). SPECT/CT imaging with ${ }^{111} \mathrm{In}$-labeled $28 \mathrm{H} 1$ as a tracer revealed a significant correlation between uptake of ${ }^{111}$ In-diethylenetriaminepentaacetic acid (DTPA)-28H1 in inflamed joints and the severity of arthritis (16). To further improve this tracer, we examined the use of ${ }^{99 \mathrm{~m}} \mathrm{Tc}$ for radiolabeling. Because of the shorter half-life of ${ }^{99 \mathrm{~m}} \mathrm{Tc}(6 \mathrm{~h})$ than of ${ }^{111}$ In $(67 \mathrm{~h})$, it is a better match with the relatively short circulation time of $28 \mathrm{H} 1$. Additionally, the radiation dose per scan will be lower, ${ }^{99 \mathrm{~m}} \mathrm{Tc}$ is less expensive, and image resolution will be higher than that with ${ }^{111}$ In-labeled tracers.

In this study, we focused on the potential role of antibody $28 \mathrm{H} 1$ as a specific and sensitive tool for early response monitoring after treatment with LCL containing PLP (PLP-LCL) in murine CIA. The CIA model is considered to be a suitable model for studying disease progression and the therapeutic effects of antirheumatic drugs.

\section{MATERIALS AND METHODS}

\section{Preparation of Liposomes}

LCL containing PLP at $2.1 \mathrm{mg} / \mathrm{mL}$ were prepared by injection of an ethanolic lipid solution into an aqueous dispersion medium and then extrusion as described earlier $(11,17)$. In brief, dipalmitoylphosphatidylcholine and 1,2-distearoyl-phosphatidylethanolaminemethyl-polyethylene glycol conjugate 2000 (both from Lipoid $\mathrm{GmbH}$ ) and cholesterol (from BUFA) (molar ratio, 1.85:0.15:1) were dissolved in ethanol. The aqueous drug solution contained PLP (BUFA) at $100 \mathrm{mg} / \mathrm{mL}$ in water for injection. The ethanolic lipid solution was injected into the aqueous solution, and the resulting coarse dispersion was downsized by multiple extrusion steps (4 or 5 for each pore size) through polycarbonate filter membranes with decreasing pore sizes of 200, 100, and $50 \mathrm{~nm}$ (Whatman Nuclepore Track-Etch Membrane; Sigma-Aldrich). Size (111 nm) and mean polydispersity (0.07) were determined by dynamic light scattering with a Malvern 4700 system (Malvern Ltd.). Nonencapsulated
PLP was removed by dialysis against saline in Slide-A-Lyzer dialysis cassettes (Thermo Fisher Scientific) with a molecular weight cutoff of $10 \mathrm{kDa}$. The dialysis medium was refreshed 4 times after at least $8 \mathrm{~h}$.

\section{Antibody Conjugation}

Monoclonal anti-FAP antibody $28 \mathrm{H} 1(3.7 \mathrm{mg} / \mathrm{mL})$ was conjugated with a 10-fold molar excess of succinimidyl-hydrazinonicotinamide (S-HYNIC) (Solulink, Inc.) in $1 \mathrm{M} \mathrm{NaHCO}_{3}(\mathrm{pH}$ 8.2) for $1 \mathrm{~h}$ at room temperature. Nonconjugated S-HYNIC was removed by dialysis against ammonium acetate $(0.25 \mathrm{M}$; pH 5.5) in Slide-A-Lyzer dialysis cassettes with a molecular weight cutoff of $10 \mathrm{kDa}$. The dialysis medium was refreshed 4 times after at least $8 \mathrm{~h}$.

\section{Radiolabeling Procedure}

$\mathrm{SnSO}_{4}(1 \mathrm{mg} / \mathrm{mL})$ was freshly prepared by adding $5 \mathrm{mg}$ of $\mathrm{SnSO}_{4}$ to $5 \mathrm{~mL}$ of $0.1 \mathrm{M} \mathrm{HCl}$. This solution was flushed with $\mathrm{N}_{2}$ (gas) for $10 \mathrm{~min}$ to prevent oxidation of $\mathrm{SnSO}_{4}$ during the labeling procedure. Tricine (100 mg per $\mathrm{mL}$ of phosphate-buffered saline; $\mathrm{pH} 7$; coligand), $0.02 \mathrm{mg}$

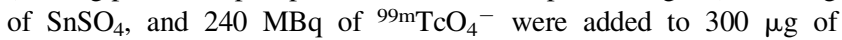
$\mathrm{S}-\mathrm{HYNIC}-28 \mathrm{H} 1$, and the mixture was incubated at room temperature for $30 \mathrm{~min}$. Radiolabeling efficiency was determined by instant thinlayer chromatography on ITLC-SG strips (Agilent Technologies) with $0.1 \mathrm{M}$ citrate ( $\mathrm{pH}$ 6.0) as the mobile phase. Labeling efficiency exceeded $95 \%$ or, if it was less than $95 \%$, then the labeled product was purified by gel filtration chromatography on a PD-10 column (GE Healthcare) to provide a preparation with a radiochemical purity of greater than $95 \%$.

\section{Animals}

Male DBA/1J mice, 10-12 wk old, were purchased from Janvier Labs. All mice were housed in filter-top cages under pathogen-free conditions. Food (SNIFF Voer) and water were available ad libitum. All animals were allowed to become accustomed to the environment for at least $1 \mathrm{wk}$ before experiments were initiated. All in vivo experiments were approved by the Animal Welfare Committee of Radboud University, Nijmegen, The Netherlands, and were conducted in accordance with the principles of the revised Dutch Act on Animal Experimentation (1997).

\section{CIA Model}

CIA was induced as described previously (18). In brief, male DBA/1J mice were immunized with $100 \mu \mathrm{g}$ of bovine type II collagen emulsified in Freund complete adjuvant (Difco Laboratories) and injected intradermally into the tail base. After $3 \mathrm{wk}$ (day 21), the mice received a booster injection of $100 \mu \mathrm{g}$ of bovine type II collagen in saline. The onset of CIA occurred a few days after the booster injection. The onset and development of arthritis were macroscopically monitored by independent observers in a masked fashion and scored on a scale from 0 to 2 for each paw. Cumulative scoring was based on redness, swelling, and ankylosis: 0, no sign of arthritis; $0.25,1$ or 2 toes red or swollen; 0.5 , 3-5 toes red or swollen; 1.0, swollen ankle; 1.5 , swollen footpad; and 2.0, severe swelling and ankylosis (16).

\section{Liposomal Treatment with PLP}

Liposomal treatment was given $1 \mathrm{~d}$ after the booster injection. The treated mice received a single intravenous injection of PLP $(10 \mathrm{mg} / \mathrm{kg})$ in LCL, with a lipid dose of $100 \mu \mathrm{mol} /$ mouse, in a total volume of $200 \mu \mathrm{L}$. The control mice received empty LCL at the same lipid dose.

\section{Small-Animal SPECT/CT Imaging and Ex Vivo Biodistribution Studies}

Treated and untreated DBA/1J mice with CIA were injected intravenously with $50 \mu \mathrm{g}$ of ${ }^{99 \mathrm{~m}} \mathrm{Tc}$-labeled S-HYNIC-28H1 (specific activity, $800 \mathrm{MBq} / \mathrm{mg}$, in $200 \mu \mathrm{L} ; n=5$ per group) at day 1,4 , or 8 after therapy. At $24 \mathrm{~h}$ after tracer injection, mice were euthanized by $\mathrm{CO}_{2} / \mathrm{O}_{2}$ 
suffocation. Subsequently, images were acquired with a U-SPECT-II/ CT scanner (MILabs) (19). Images were acquired as 2 frames of $25 \mathrm{~min}$ with a $1.0-\mathrm{mm}$-diameter cylindrical pinhole mouse ultra-high-sensitivity collimator. SPECT scans were followed by CT scans for anatomic referencing (spatial resolution, $160 \mu \mathrm{m} ; 65 \mathrm{kV} ; 615 \mu \mathrm{A}$ ). Scans were reconstructed with MILabs reconstruction software (which uses an ordered-subset expectation maximization algorithm), a voxel size of $0.2 \mathrm{~mm}, 16$ subsets, and 1 iteration.

After image acquisition, relevant tissues were dissected to determine the accumulation of the tracer in the organs and the joints. Tissues of interest (right wrist joint, left wrist joint, right ankle joint, and left ankle joint) were dissected and weighed, and radioactivity was measured in a shielded well-type $\gamma$-counter ( $\sim 7.6 \mathrm{~cm}[3$ in]; Perkin-Elmer). Injection standards $(1 \%)$ were counted simultaneously with tissue samples to correct for physical decay and to allow calculation of the percentage injected dose (\%ID) per gram of tissue $(\% \mathrm{ID} / \mathrm{g})$.

\section{Quantitative SPECT Analysis}

Reconstructed micro-SPECT scans were coregistered with CT images. A gaussian filter of 0.8 was applied to the SPECT images, and a 3-dimensional volume of interest was drawn around the arthritic joints with Inveon Research Workplace 4.1 software (Siemens Preclinical Solutions). Voxel intensity values were converted to \% ID with decay correction and a standard curve (voxel intensity vs. $\mathrm{kBq}$ ) acquired by scanning and reconstructing known ${ }^{99 \mathrm{~m} T c}$ activities from 0 to 370 $\mathrm{kBq}$ under the same conditions as those used during the animal scans.

\section{Statistical Analysis}

Statistical analysis was performed with GraphPad Prism version 5.03 for Windows (GraphPad Software). Values are reported as mean \pm SD. The data were analyzed with nonparametric Mann-Whitney $U$ tests. All tests were 2-sided, and $P$ values of less than 0.05 were considered to be significant.

\section{RESULTS}

\section{PLP-LCL Therapy}

Treatment with a single injection of PLP-LCL at $10 \mathrm{mg} / \mathrm{kg}$ was started after the onset of arthritis and was effective, as macroscopic arthritis scores were lower in treated mice than in control mice (Fig. 1). Visual arthritis scores were measured on a scale from 0 to 2 per paw and from 0 to 8 per mouse until $9 \mathrm{~d}$ after therapy. Arthritis scores were significantly lower in the treated group than in the control group at all time points. At day 2 after treatment, the arthritis scores had decreased in treated mice but had increased in control mice $(P=0.01)$. This effect was still seen at $5 \mathrm{~d}$ after treatment $(P=0.01)$ and at $9 \mathrm{~d}$ after treatment $(P=0.02)$.

\section{SPECT/CT Imaging}

Inflamed joints were specifically visualized by SPECT/CT

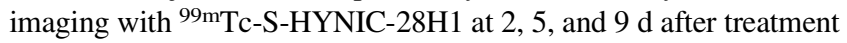
(Fig. 2). Images clearly showed preferential uptake of the anti-FAP antibody in inflamed paws, with low background accumulation in other tissues; even joints with minimal arthritis scores $(0.25$ on a scale of 0-2) were delineated. Increased uptake of the radiolabeled anti-FAP antibody in inflamed joints of control mice was shown over time (Figs. 2A-2C), whereas the joints of treated mice did not show enhanced uptake (Figs. 2D-2F).

\section{Quantitative SPECT Analysis}

Tracer accumulation in each paw on SPECT/CT images was analyzed quantitatively. Analysis showed that tracer accumulation was lower in treated mice than in control mice and was significantly lower at 5 and $9 \mathrm{~d}$ after treatment $(P<0.02)$ (Fig. 3A). Tracer

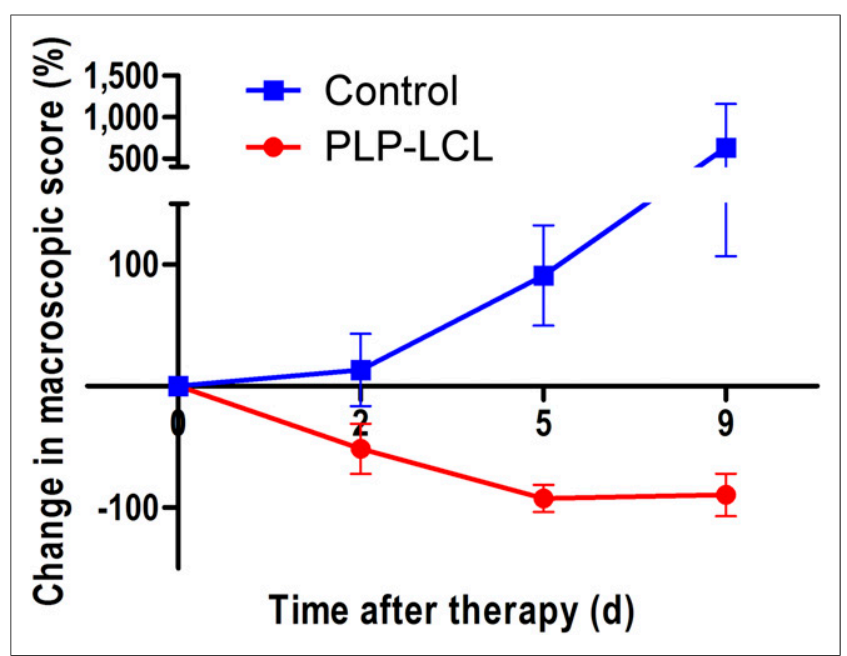

FIGURE 1. Changes in macroscopic arthritis scores over time after single treatment with PLP-LCL at $10 \mathrm{mg} / \mathrm{kg}$ (red circles) vs. empty LCL (blue squares). Values are mean \pm SD for 5 mice per group. Mann-Whitney $U$ tests showed that differences between control and treated animals were significant at all time points $(P=0.01, P=0.01$, and $P=0.02$ at 2,5 , and $9 \mathrm{~d}$ after treatment, respectively).

accumulation in joints of treated mice was $4.1 \pm 3.5,1.9 \pm 1.0$, and $3.2 \pm 1.1 \% \mathrm{ID} / \mathrm{g}$ at 2,5 , and $9 \mathrm{~d}$ after treatment, respectively; for untreated mice, the corresponding values were $3.5 \pm 3.0,3.1 \pm 2.8$, and $6.9 \pm 4.1 \% \mathrm{ID} / \mathrm{g}$ at 2,5 , and $9 \mathrm{~d}$ after injection of empty LCL, respectively. A good correlation was found between the values obtained for ${ }^{99 \mathrm{~m}} \mathrm{Tc}-\mathrm{S}-\mathrm{HYNIC}-28 \mathrm{H} 1$ uptake in the quantitative SPECT analysis and the arthritis scores (Spearman $\rho, 0.6830$; $P<0.0001)$ (Fig. 3B).

\section{Biodistribution Studies}

Measurement of the activity concentration in dissected organs showed that the accumulation of ${ }^{99 m}$ Tc-S-HYNIC-28H1 in joints was lower in arthritic mice treated with PLP-LCL than in control mice $(P=0.01)$ (Fig. 4A). Tracer accumulation in joints of treated mice was $5.9 \pm 3.1,3.3 \pm 1.4$, and $4.1 \pm 1.2 \% \mathrm{ID} / \mathrm{g}$ at 2,5 , and $9 \mathrm{~d}$ after treatment, respectively. These values were lower than those for

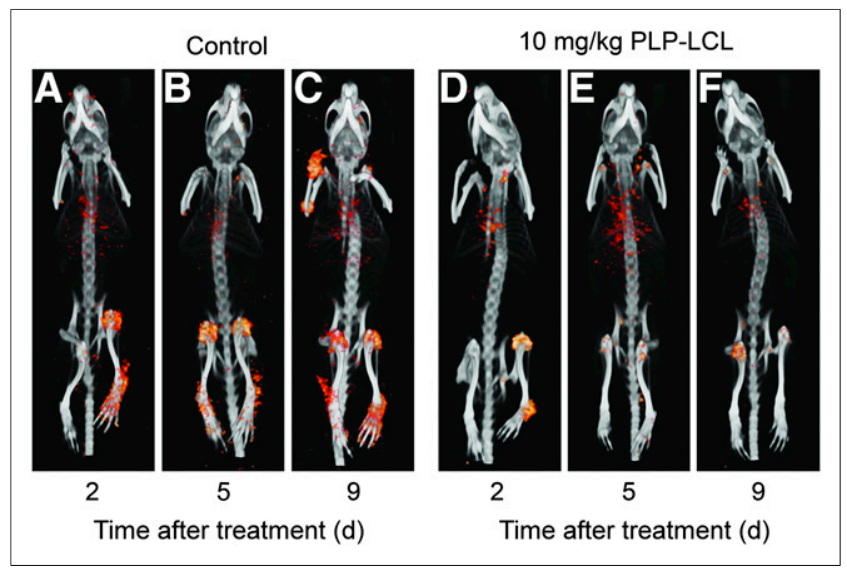

FIGURE 2. 3-dimensional SPECT/CT scans of control (A-C) and PLP-

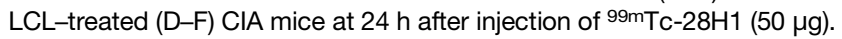
Image acquisition was performed $2 \mathrm{~d}(\mathrm{~A}$ and $\mathrm{D}), 5 \mathrm{~d}(\mathrm{~B}$ and $\mathrm{E})$, and $9 \mathrm{~d}(\mathrm{C}$ and F) after therapy. All images are scaled equally. 


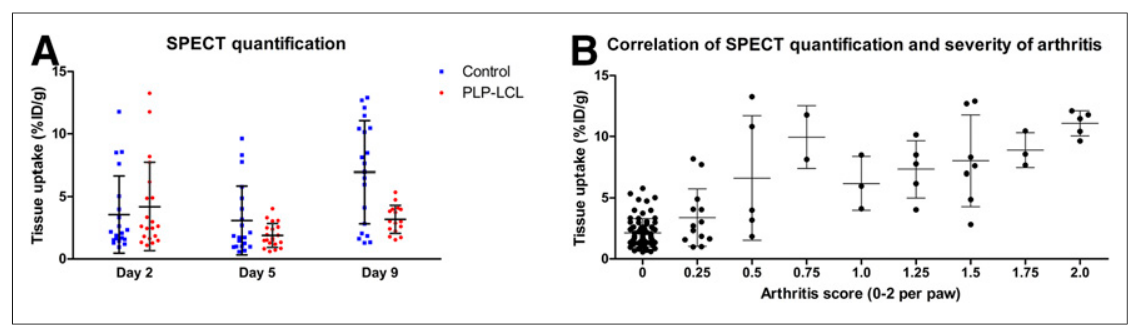

FIGURE 3. (A) Tissue uptake-based quantitative SPECT analysis at 2, 5, and $9 \mathrm{~d}$ after treatment with PLP-LCL at $10 \mathrm{mg} / \mathrm{kg}$. Mann-Whitney $U$ tests showed significantly lower levels of tissue uptake after treatment with PLP-LCL than in control group $(P=0.02)$. (B) Correlation between tissue uptake of $99 \mathrm{mTC}$-S-HYNIC-28H1 at $24 \mathrm{~h}$ after injection and arthritis scores (Spearman $\rho$, 0.6830; $P<0.0001)$. Tissue uptake (\% ID/g) per individual paw ( 4 or 5 mice per group, 4 joints per mouse) is shown.

control mice, in which tracer accumulation in joints was $7.2 \pm 5.2$, $5.1 \pm 4.2$, and $11.7 \pm 5.6 \% \mathrm{ID} / \mathrm{g}$ at 2,5 , and $9 \mathrm{~d}$ after injection of empty LCL, respectively. Whole-body distribution of radioactivity in treated mice did not differ significantly from that in control mice at all time points (data not shown). Additionally, in line with the quantitative SPECT analysis, a correlation was found between the values obtained for ${ }^{99 m} \mathrm{Tc}-\mathrm{S}-\mathrm{HYNIC}-28 \mathrm{H} 1$ uptake in the biodistribution studies and the arthritis scores (Spearman $\rho$, 0.6699; $P<$ 0.0001 ) (Fig. 4B). Furthermore, the values obtained for ${ }^{99 m} \mathrm{Tc}-\mathrm{S}-$ HYNIC-28H1 uptake in the biodistribution studies correlated with the uptake values found in the quantitative SPECT analysis $(r$, 6.072; $P<0.0001)$.

\section{DISCUSSION}

The present study demonstrated that SPECT images at $24 \mathrm{~h}$ after injection of a ${ }^{99 m}$ Tc-labeled anti-FAP antibody reflected the severity of arthritis and therapeutic efficacy $(6,11,12,20)$. These data indicated that ${ }^{99 \mathrm{~m}} \mathrm{Tc}-\mathrm{S}-\mathrm{HYNIC}-28 \mathrm{H} 1$ could be used for noninvasive monitoring of the therapeutic effects of PLP-LCL in a mouse model of arthritis. Our previous study showed that the uptake of ${ }^{111} \mathrm{In}-$ DTPA-28H1 in inflamed joints was specific and correlated with the severity of arthritis and that ${ }^{111}$ In-DTPA-28H1 SPECT/CT resulted in high-quality images (16). The data indicated that molecular imaging with an anti-FAP antibody might provide added value for early and sensitive response monitoring.

Ideally, multiple images would be acquired within a short period of time, just before therapy and early after therapy. To allow for this schedule, repeated injections of the tracer would be necessary. High-quality images were obtained with ${ }^{111}$ In-DTPA-28H1, and the half-life $(2.8 \mathrm{~d})$ of ${ }^{111} \mathrm{In}$ is suitable for antibody imaging but too long to allow repeated imaging of the same patient within a short period. Therefore, we radiolabeled $28 \mathrm{H} 1$ with a radionuclide that has a relatively short physical half-life. With a half-life of $6 \mathrm{~h}$, the radionuclide ${ }^{99 \mathrm{~m}} \mathrm{Tc}$ is potentially suitable for repeated imaging at multiple time points at short intervals. To allow efficient and stable labeling of $28 \mathrm{H} 1$ with ${ }^{99 \mathrm{~m}} \mathrm{Tc}$, we conjugated the antibody with SHYNIC and labeled it in the presence of tricine as a coligand.

Because $28 \mathrm{H} 1$ is a humanized antibody that would induce a severe immune response with repeated exposure in mice, we were not able to image the mice repeatedly and monitor the therapeutic effects in the same animal. However, here we showed that ${ }^{99 \mathrm{~m}} \mathrm{Tc}-\mathrm{S}$ HYNIC-28H1 might be used to monitor the therapeutic effects in RA patients at multiple time points. The potential ability of our construct to monitor therapeutic efficacy was examined with PLPLCL therapy because it has been shown to result in rapid, strong therapeutic benefits in experimental RA models $(6,11,12,20)$. The therapeutic effects in mice were monitored by visual arthritis scoring; the scores had already decreased $2 \mathrm{~d}$ after the administration of PLP-LCL, and almost complete remission of macroscopic signs of arthritis was observed at least until $9 \mathrm{~d}$ after treatment (Fig. 1).

With PLP treatment, lower levels of tumor necrosis factor $\alpha$ and interleukin 1 are produced in inflamed joints, in turn leading to decreased activation of fibroblastlike synoviocytes, resulting in reduced production of FAP $(9,10)$. We hypothesized that this effect

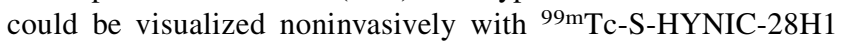
SPECT/CT. High image quality was obtained, and the activity of the radiolabeled antibody in background tissue was low (Fig. 2). Over time, images showed increasing uptake in the joints of mice in the control group and decreasing uptake in the joints of mice that received PLP-LCL; these findings demonstrated that the therapeutic effects of PLP-LCL could be visualized noninvasively with ${ }^{99 \mathrm{~m}} \mathrm{Tc}-$ S-HYNIC-28H1 SPECT/CT.

The findings were confirmed after quantification of the SPECT images (Fig. 3A) and by the ex vivo biodistribution studies (Fig. 4A). In both analyses, values correlated with macroscopic arthritis scores (Figs. 3B and 4B); this correlation was also observed with the ${ }^{111}$ In-labeled compound (16). The correlation found between macroscopic arthritis scores and ex vivo biodistribution data was lower than that found between macroscopic arthritis scores and quantitative imaging.

Significant uptake of ${ }^{99 \mathrm{~m}} \mathrm{Tc}-\mathrm{S}-\mathrm{HYNIC}$ $28 \mathrm{H} 1$ was observed in multiple joints of individual mice; these joints were not considered in our macroscopic arthritis scoring system, so the uptake of ${ }^{99 \mathrm{~m}} \mathrm{Tc}$ S-HYNIC-28H1 resulted in a more complete representation of the severity of disease. Even paws with low arthritis scores were visualized by ${ }^{99 m}$ Tc-S-HYNIC- $28 \mathrm{H} 1$ SPECT, although the joints did not show 
obvious macroscopic symptoms; these findings indicated the potentially high sensitivity of this imaging method for monitoring the onset of disease. These data suggest that this imaging method outperforms the macroscopic arthritis scoring method for identifying mildly involved joints and can measure inflammation in joints that cannot be scored macroscopically.

Quantitative SPECT analysis at day 9 after therapy can also support the hypothesis of high sensitivity of imaging with ${ }^{99 \mathrm{~m}} \mathrm{Tc}-$ S-HYNIC-28H1. The arthritis scores did not increase in the treated group, whereas the SPECT values increased, although not significantly. This finding can indicate an early decrease in the effect of PLP that was not yet detected macroscopically, emphasizing that molecular imaging may detect flare-ups at earlier time points than macroscopic arthritis scoring. However, we cannot rule out the possibility that the increase in tissue uptake was a result of variations between the groups. The mice that were imaged at day 5 were not the same as those that were imaged at day 9. The mice were divided into groups on the basis of macroscopic arthritis scores at the start of therapy, but because of the systemic and progressive character of the model, the groups were not equally affected at the time of imaging. Although no significant differences were found in macroscopic arthritis scores between the mice in the day 5 group and those in the day 9 group at the start of therapy, the differences in tissue uptake could be explained by slightly more severely affected mice in the day 9 group.

\section{CONCLUSION}

99mTc-S-HYNIC-28H1 SPECT is a more specific and potentially a more sensitive method for the early detection of exacerbations in RA and for therapy monitoring, relative to the arbitrary macroscopic arthritis scoring system. Therefore, this reliable, noninvasive method may be valuable for immediate therapy monitoring and further therapy planning in RA patients, with the aim of improving individual treatment strategies.

\section{DISCLOSURE}

This study was supported by a grant from NanoNextNL Drug Delivery Program 03D.06. No other potential conflict of interest relevant to this article was reported.

\section{ACKNOWLEDGMENTS}

We thank Bianca Lemmers-van de Weem, Sharon Wennekers, Iris Lamers-Elemans, and Kitty Lemmens-Hermans for their technical assistance in the animal experiments.

\section{REFERENCES}

1. Smolen JS, Steiner G. Therapeutic strategies for rheumatoid arthritis. Nat Rev Drug Discov. 2003;2:473-488.

2. Smolen JS, Landewe R, Breedveld FC, et al. EULAR recommendations for the management of rheumatoid arthritis with synthetic and biological diseasemodifying antirheumatic drugs: 2013 update. Ann Rheum Dis. 2014;73:492-509.

3. Caporali R, Scire CA, Todoerti M, Montecucco C. Glucocorticoids in rheumatoid arthritis. Drugs. 2013;73:31-43.

4. Firestein GS. Invasive fibroblast-like synoviocytes in rheumatoid arthritis: passive responders or transformed aggressors? Arthritis Rheum. 1996;39:1781-1790.

5. Kinne RW, Brauer R, Stuhlmuller B, Palombo-Kinne E, Burmester GR. Macrophages in rheumatoid arthritis. Arthritis Res. 2000;2:189-202.

6. Ozbakir B, Crielaard BJ, Metselaar JM, Storm G, Lammers T. Liposomal corticosteroids for the treatment of inflammatory disorders and cancer. $J$ Control Release. 2014;190:624-636.

7. Bartok B, Firestein GS. Fibroblast-like synoviocytes: key effector cells in rheumatoid arthritis. Immunol Rev. 2010;233:233-255.

8. Bauer S, Jendro MC, Wadle A, et al. Fibroblast activation protein is expressed by rheumatoid myofibroblast-like synoviocytes. Arthritis Res Ther. 2006;8:R171.

9. Metselaar JM, van den Berg WB, Holthuysen AE, Wauben MH, Storm G, van Lent PL. Liposomal targeting of glucocorticoids to synovial lining cells strongly increases therapeutic benefit in collagen type II arthritis. Ann Rheum Dis. 2004;63:348-353.

10. Russell A, Haraoui B, Keystone E, Klinkhoff A. Current and emerging therapies for rheumatoid arthritis, with a focus on infliximab: clinical impact on joint damage and cost of care in Canada. Clin Ther. 2001;23:1824-1838.

11. van der Geest T, Metselaar JM, Gerrits D, et al. ${ }^{18}$ F-FDG PET/CT imaging to monitor the therapeutic effect of liposome-encapsulated prednisolone in experimental rheumatoid arthritis. J Control Release. 2015;209:20-26.

12. Metselaar JM, Wauben MH, Wagenaar-Hilbers JP, Boerman OC, Storm G. Complete remission of experimental arthritis by joint targeting of glucocorticoids with long-circulating liposomes. Arthritis Rheum. 2003;48:2059-2066.

13. Rosado-de-Castro PH, Lopes de Souza SA, Alexandre D, Barbosa da Fonseca LM, Gutfilen B. Rheumatoid arthritis: nuclear medicine state-of-theart imaging. World J Orthop. 2014;5:312-318.

14. Ibrahim SM, Koczan D, Thiesen HJ. Gene-expression profile of collageninduced arthritis. J Autoimmun. 2002;18:159-167.

15. Crielaard BJ, Lammers T, Schiffelers RM, Storm G. Drug targeting systems for inflammatory disease: one for all, all for one. J Control Release. 2012;161:225-234.

16. Laverman P, van der Geest T, Terry SY, et al. Immuno-PET and immuno-SPECT of rheumatoid arthritis with radiolabeled anti-fibroblast activation protein antibody correlates with severity of arthritis. J Nucl Med. 2015;56:778-783.

17. Anderson R, Franch A, Castell M, et al. Liposomal encapsulation enhances and prolongs the anti-inflammatory effects of water-soluble dexamethasone phosphate in experimental adjuvant arthritis. Arthritis Res Ther. 2010;12:R147.

18. Joosten LA, Helsen MM, van de Loo FA, van den Berg WB. Anticytokine treatment of established type II collagen-induced arthritis in DBA/1 mice: a comparative study using anti-TNF alpha, anti-IL-1 alpha/beta, and IL-1Ra. Arthritis Rheum. 1996;39:797-809.

19. van der Have F, Vastenhouw B, Ramakers RM, et al. U-SPECT-II: an ultra-highresolution device for molecular small-animal imaging. J Nucl Med. 2009;50:599-605.

20. van den Hoven JM, Hofkens W, Wauben MH, et al. Optimizing the therapeutic index of liposomal glucocorticoids in experimental arthritis. Int J Pharm. 2011;416:471-477. 